International Journal of Engineering \& Technology, 7 (4.10) (2018) 992-994
International Journal of Engineering \& Technology
SPC
Website: www.sciencepubco.com/index.php/IJET
Research paper

\title{
A Discourse on the Estimation of Nonlinear Regression Model
}

\author{
B. Mahaboob ${ }^{1}$, B.Venkateswarlu ${ }^{2}$, J. Ravi Sankar ${ }^{3}$, J. PeterPraveen ${ }^{4}$ and C. Narayana ${ }^{5}$ \\ ${ }^{1,4}$ Department of Mathematics, K.L.E.F (Deemed to be University), Vaddeshwaram, Vijayawada, Andhra Pradesh. \\ ${ }^{2,3}$ Department of Mathematics, VIT University, Vellore, Tamilnadu. \\ ${ }^{5}$ Department of Mathematics, Sri Harsha institute of P.G Studies, Nellore. \\ *Corresponding author E-mail: venkatesh.reddy@vit.ac.in
}

\begin{abstract}
The present study evaluates an estimation for regression model which are nonlinear with Goldfeld, Quandt and exponential structure for heteroscedastic errors. An IENLGLS (Iterative Estimated Nonlinear Generalised Least Squares) estimator based on Goldfeld and Quandt for parametric vector has been derived in this research article. Volkan Soner Ozsoy e.t.al [1], in their paper, proposed an effective approach based on the particle Swarm Optimisation (PSO) algorithm in order to enhance the accuracy in the estimation of parameters of nonlinear regression model. Ting Zhang et.al [2], in their article, established an asymptotic theory for estimates of the time-varying regression functions. Felix Chan et.al [3], in their paper, proposed some principals which are sufficient for asymptotic normality and consistency of the MLH estimator
\end{abstract}

Keywords: Nonlinear regression model, Heteroscedastic error, nonlinear internally studentized residuals, OLS (Ordinary Least Squares), Regressor matrix.

\section{Introduction}

The advent of modern computer science has made it possible for the applied mathematician to study the inferential aspects of an increasing number of nonlinear models in recent years. In Applied Mathematics, one may deal with nonlinearity in many diverse ways. For instance nonlinearity appears in parameters but not in variables in most of the time series and distributed log models. In Mathematical Economics, more general nonlinear regression models are used in the estimation of production functions and demand functions. The Cobb-Douglas production function involving an additive error term; Constant Elasticity of Substitution (CES) and Variable Elasticity of Substitution (VES) production functions are more complicated and highly nonlinear models. Generally a nonlinear model refers to regression function which is nonlinear either in predictor variables or in the unknown parameters or in both predictor variables and parameters. Nonlinear models can be broadly classified into two parts namely (A) Non linear models which are nonlinear in regressors but linear in parameters.

(B) Nonlinear models which are which nonlinear in parameters. Nonlinear regression analysis is a powerful method for analysing the data described by models which are non linear in parameters. Generally a researcher has mathematical expression, which relates the dependent variable to the independent variables and these models are nonlinear in parameters. Under these cases usually the linear regression analysis can be extended which introduces considerable complexity. A more common problem with data that are best fit by a nonlinear regression model than with data that can be adequately fit by the linear regression model is the problem of Heteroscedasticity of errors. For instance it may arise in data from pharmacokinetics and chemical reactions. The heteroscedastic phenomenon can be discussed in many ways with respect to nonlinear regression models. In the present study, an attempt has been made by developing inferential methods for heteroscedastic nonlinear regression analysis.

\section{Estimation of nonlinear regression model with goldfeld and quandt structure for heteroscedastic errors}

Consider a standard nonlinear regression model with the heteroscedastic structure for errors suggested by Goldfeld and Quandt (1972) as

$Y_{i}=f\left(X_{i}, \beta\right)+\varepsilon_{i}, 1,2, \ldots n$

Or

$Y_{n \times 1}=f_{n \times 1}\left(\beta_{n \times 1}\right)+\varepsilon_{n \times 1}$

such that $\mathrm{E}[\varepsilon]=\mathrm{O}$ and $\mathrm{E}\left(\varepsilon \varepsilon^{\prime}\right)=\Phi=\operatorname{diag}\left(\sigma_{1}^{2}, \sigma_{2}^{2}, \ldots, \sigma_{\mathrm{n}}^{2}\right)$ Assume $\sigma_{\mathrm{i}}^{2}=\mathrm{Z}_{\mathrm{i}}^{\prime} \delta$. Where $\mathrm{Z}_{\mathrm{i}}$ is (kx 1$)$ vector of known observations on certain independent variables $\mathrm{X}_{\mathrm{i}}{ }^{\prime} \mathrm{s}$; and $\delta$ is a (k x 1$)$ vector of unknown parameters. One may obtain an estimator for $\delta$ by regressing linearly the squares of iterative nonlinear internally studentized residuals $\left(\mathrm{e}_{\mathrm{ni}}^{*^{2}}\right)$ on $\mathrm{Z}_{\mathrm{i}}$. Thus, the regression model may be written as

$\mathrm{e}_{\mathrm{n}}^{*}=\mathrm{Z} \delta+\mathrm{W}$ 
Where $\dot{\mathrm{e}}_{\mathrm{n}}:(\mathrm{n} \times 1)$ vector of $\mathrm{e}_{\mathrm{ni}}^{*^{2}}, \mathrm{Z}$ is a matrix of order $\mathrm{nxk}$ whose entries are known.$\delta$ is column vector whose entries are unknown and they are heteroscedastic error variables. The elements of $\delta$ are parameters , W: (n x 1) vector of well-behaved error observations.

The ordinary least squares (OLS) estimator of $\delta$ is given by

$\delta_{\mathrm{n}}^{*}=\left(\mathrm{Z}^{\prime} \mathrm{Z}\right)^{-1} \mathrm{Z}^{\prime} \mathrm{e}_{\mathrm{n}}^{*}$

Now, estimators for $\sigma_{i}^{2}$ and $\Phi$ are respectively given by

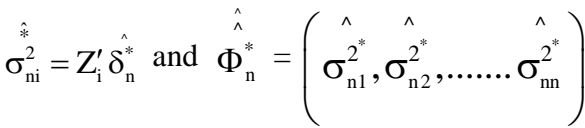

An Iterative Estimated Nonlinear Generalized Least Squares (IENLGLS) estimator based on Goldfeld and Quandt structure for $\beta$ is given by

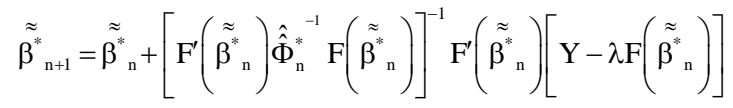

Here, $\quad F\left({\tilde{\beta^{*}}}^{*}\right)=\left[\frac{\partial \mathrm{f}}{\partial \beta^{\prime}}\right]_{\tilde{\beta}^{*}{ }_{n}}$ as the regressor matrix and $\left[\mathrm{Y}-\lambda \mathrm{f}\left({\tilde{\beta^{*}}}_{\mathrm{n}}\right)\right]$ is the vector of IENLGLS residuals based on Goldfeld and Quandt structure for an arbitrary value of $\lambda$.

Further, $\operatorname{var}\left({\tilde{\beta^{*}}}_{\mathrm{n}}^{*}\right)=\left[\mathrm{F}^{\prime}\left({\tilde{\beta^{*}}}_{\mathrm{n}}^{*}\right) \hat{\hat{\Phi}}_{\mathrm{n}}^{*^{-1}} \mathrm{~F}\left({\tilde{\beta^{*}}}_{\mathrm{n}}\right)\right]^{-1}$

\section{Estimation of nonlinear regression model with exponential structure for heteroscedastic errors}

Consider a standard nonlinear regression model with a heteroscedastic error structure, where the error variance is exponentially related to the expectation of the dependent variable as

$$
Y_{i}=f\left(X_{i}, \beta\right)+\varepsilon_{i}, i=1,2, \ldots, n
$$

(or)

$$
\mathrm{Y}_{\mathrm{n} \times 1}=\mathrm{f}_{\mathrm{n} \times 1}\left(\beta_{\mathrm{p} \times 1}\right)+\varepsilon_{\mathrm{n} \times 1}
$$

Such that $\mathrm{E}\left(\mathrm{Y}_{\mathrm{i}}\right)=\mathrm{f}\left(\mathrm{X}_{\mathrm{i}}, \beta\right)$ or $\mathrm{E}\left(\varepsilon_{\mathrm{i}}\right)=0$ and

$\operatorname{Var}\left(Y_{i}\right)=\sigma^{2}\left[f\left(X_{i}, \beta\right)\right]^{\delta}=\sigma_{i}^{2}$ (say)

or $\operatorname{Var}\left(\varepsilon_{\mathrm{i}}\right)=\sigma_{\mathrm{i}}^{2}=\sigma^{2}\left[\mathrm{f}\left(\mathrm{X}_{\mathrm{i}}, \beta\right)\right]^{\delta}, \mathrm{i}=1,2, \ldots \mathrm{n}$.

Also $\varepsilon_{\mathrm{i}}$ 'S are independently and normally distributed errors. Here, $\sigma^{2}$ and $\delta$ are unknown scalar heteroscedastic parameters. Consider Iterative Nonlinear Internally Studentized residuals as $\mathrm{e}_{\mathrm{ni}}^{*}$ based on $\hat{\beta}_{\mathrm{n}}$. Rewrite the equation (3.3) as $\log \sigma_{i}^{2}=\log \sigma^{2}+\delta \log f\left(X_{i}, \beta\right), i=1,2, \ldots, n$.

Replacing $\sigma_{i}^{2}$ by $e_{n i}^{*^{2}}, \beta$ by $\hat{\beta}_{n}$ and introducing a well behaved error term $\mathrm{W}_{\mathrm{i}}$, equation (3.4) can be written as

$\log \mathrm{e}_{\mathrm{ni}}^{*^{2}}=\log \sigma^{2}+\delta \log \mathrm{f}\left(\mathrm{X}_{\mathrm{i}}, \hat{\beta}_{\mathrm{n}}\right)+\mathrm{W}_{\mathrm{i}}, \mathrm{i}=1,2, \ldots \mathrm{n}$

Now, the OLS estimators of $\sigma^{2}$ and $\delta$ can be obtained as $\hat{\sigma}^{2}$ and $\hat{\delta}$ and an estimator for $\sigma_{i}^{2}$ is given by

${\overline{\sigma_{\mathrm{ni}}}}_{*_{2}}^{x^{2}} \overline{\sigma^{2}}\left[\mathrm{f}\left(\mathrm{X}_{\mathrm{i}}, \hat{\beta}_{\mathrm{n}}\right)\right]^{\hat{\delta}}$

Also, an estimator for heteroscedastic error variance-covariance matrix is given by

$\bar{\Phi}_{\mathrm{n}}^{*}=\operatorname{diag}\left(\overline{\sigma_{\mathrm{n} 1}^{*_{2}}}, \overline{\sigma_{\mathrm{n} 2}^{*_{2}}}, \ldots \overline{\sigma_{\mathrm{nn}}^{*_{2}}}\right)$

Hence, an Iterative Estimated Nonlinear Generalized Least Squares (IENLGLS) estimator for $\beta$ based on exponential heteroscedastic error structure is given by

$\overline{\beta_{\mathrm{n}+1}^{*}}=\overline{\beta_{\mathrm{n}}^{*}}+\left[\mathrm{F}^{\prime}\left(\overline{\beta_{\mathrm{n}}^{*}}\right) \Phi_{\mathrm{n}}^{*{ }^{-1}} \mathrm{~F}\left(\overline{\beta_{\mathrm{n}}^{*}}\right)\right]^{-1} \mathrm{~F}^{\prime}\left(\overline{\beta_{\mathrm{n}}^{*}}\right)\left[\mathrm{Y}-\lambda \mathrm{F}\left(\overline{\beta_{\mathrm{n}}^{*}}\right)\right]$

Here, $\quad F\left(\overline{\beta_{\mathrm{n}}^{*}}\right)=\left[\frac{\partial \mathrm{f}}{\partial \beta^{\prime}}\right]_{\beta_{\mathrm{n}}^{*}}$ as the regression matrix and $\left[\mathrm{Y}-\lambda \mathrm{f}\left(\beta_{\mathrm{n}}^{*}\right)\right]$ is the vector of IENLGLS residuals based on exponential heteroscedastic structure for an arbitrary value of $\lambda$. Further,

$\operatorname{Var}\left(\overline{\beta_{\mathrm{n}}^{*}}\right)=\left[\mathrm{F}^{\prime}\left(\overline{\beta_{\mathrm{n}}^{*}}\right) \bar{\Phi}_{\mathrm{n}}^{*} \mathrm{~F}\left(\overline{\beta_{\mathrm{n}}^{*}}\right)\right]^{-1}$

\section{Conclusion}

In this discussion, IENLGLS estimator based on Goldfeld and Quandt structure for parametric vector has been proposed. In addition to this IELGLS estimator for parametric vector based on exponential heteroscedastic error structure is presented in this study.

\section{References}

[1] Volkan Soner Ozsoy, H. Hasan ORKCU, "Estimating the parameters of nonlinear regression models", Gazi University Journal Science, 29, 1, (2016), Pp: 187-199

[2] Ting Zhang, Wei Biao Wu, "Time-Varying nonlinear regression models: Non parametric estimation and model selection", The Annals of statistics, Institute of Mathematical statistics, Vol. (43), (2015), Pp: 741-768

[3] Felix Chan, Marcelo C. Medeiros, "Structure and asymptotic theory for nonlinear models with GARCH errors", Science Direct, Vol. (16), Issue 1, (2015), Pp: 1-21.

[4] Gordon K. Smyth, "Nonlinear regression, Encyclopedia of Environmetrics Vol. (3), (2002). Pp: 1405-1411.

[5] Gurleen K. Popli , "A note on the instrumental variable estimators in the nonlinear models", Journal of Quantitative Economics Vol.16 no.2, (2000), Pp: 31-36.

[6] Davidian M. and Giltinon D.M (2003), "Nonlinear models for repeated measurement Data: An overview and update", Journal of Agricultural, Biological and Environmental statistics (JABES), Vol. (8), (2003), Pp: 387-419.

[7] Vasilyev D.M, "Theoretical and Practical Aspects of linear and nonlinear models order reduction Techniques", MIT, USA, (2008). 
[8] E. Grafarent and J. Awange, "Application of linear and nonlinear models", Springer Geophysics, (2012).

[9] Bates D.M and Walts D.G, "Nonlinear regression: Iterative Estimation and Linear Approximations in Nonlinear regression Analysis and its Applications", John Wiley and sons Inc Hobeken, NJ, USA, (2008). 